\title{
Synthesis and Characterization of Fe-ZIF-8 by Utilizing Waste Filtrate as Solvent
}

\author{
Rahmatul Fajri*, Yulida Amri \\ Department of Chemistry, Faculty of Engineering \\ Samudra University \\ Langsa, Indonesia \\ *rahmatulfajri@unsam.ac.id, yulidaamri@unsam.ac.id
}

\author{
Ratna Ediati \\ Department of Chemistry, Faculty of Mathematics and \\ Natural Sciences \\ Institute of Technology of Sepuluh Nopember (ITS) \\ Surabaya, Indonesia \\ ratnaediati@gmail.com
}

\begin{abstract}
Fe-ZIF-8 has been successfully synthesized using filtrate as a solvent with two repetitions. The temperature used in the synthesis of $\mathrm{Fe}-\mathrm{ZIF}-8$ was room temperature $\left(25-30^{\circ} \mathrm{C}\right)$, with a molar ratio of $\mathrm{Zn}(\mathrm{NO3}) 2.4 \mathrm{H} 2 \mathrm{O}$ and 2-methylimidazole $=1: 4$, while $\mathrm{FeSO} 4.7 \mathrm{H} 2 \mathrm{O}$ added was $5 \%$ of the amount of $\mathrm{Zn}$ (NO3)2.4H2O. The resulting samples were analyzed using XRD and FTIR. The results of the XRD analysis showed that the synthesized Fe-ZIF-8.1 did not have different peaks, compared to the ZIF-8 material. FTIR spectra of Fe-ZIF-8.1 which was synthesized using filtrate also did not have different absorption peaks from ZIF-8, although there were some peaks that had shifted. It indicates that Fe had been doped in ZIF-8 material to replace some of the $\mathrm{Zn}$ metal. The use of filtrate as a solvent is one effort to save the environment from the dangers of chemical waste.
\end{abstract}

Keywords-Fe-ZIF-8, waste filtrate, solvent

\section{INTRODUCTION}

$\mathrm{ZIF}$ is a porous material. It also has chemical functions in various fields, including gas storage and adsorbent [1]. ZIF has a skeleton structure constructed from various metals or metal clusters with various organic ligands. The skeleton structure of ZIF is tetrahedral, similar to the zeolite skeleton structure [2]. The difference between ZIF and zeolite lies in the position of the silicon or aluminum in the zeolite structure being replaced by transition metal ions such as $\mathrm{Zn}$ (II) or Co (II) in ZIF. In addition, the oxygen bridging $\mathrm{Si}$ and $\mathrm{Al}$ is replaced with an imidazole ligand, so that ZIF has a more flexible hybrid skeleton structure [3-5].

One type of ZIF that continues to be developed both as a catalyst and adsorbent is ZIF-8. The ZIF-8 is formed from $\mathrm{Zn}^{2+}$ and 2-methylimidazole which are tetrahedral coordinated with the empirical formula $\mathrm{Zn}$ (methylimidazole) $)_{2}$ and has a structure similar to sodalite. ZIF-8 has a pore diameter of 3.4 $\AA$, surface area reaches $1947 \mathrm{~m}^{2} / \mathrm{g}$, pore volume reaches 0.663 $\mathrm{m}^{3} / \mathrm{g}$ and is stable up to the heat of $550^{\circ} \mathrm{C}[6,7]$. It is an advantage of the ZIF-8 compared to other ZIFs for use in various applications. ZIF- 8 has been used as a gas storage, adsorbent, heterogeneous catalyst and chemical sensor [3]. The performance of ZIF-8 can be improved by adding other metal ions, such as $\mathrm{Ni}, \mathrm{Co}$ and $\mathrm{Fe}$ [8-10]. The use of $\mathrm{Fe}$ in the synthesis of ZIF-8 as an adsorbent has been successfully applied to the Remazol Deep Black RGB type pollutants found in water. The study results show that the Fe-ZIF- 8 has better capacity when compared to ZIF-8 [10].

The excessive use of solvents in the ZIF-8 synthesis method creates new problems for the environment, namely the occurrence of environmental pollution caused by waste from the synthesized filtrate. To overcome this, the researchers reduced the use of organic solvents in each stage of the synthesis, namely by recycling the remaining solvent from the ZIF-8 synthesis [11]. Another alternative is to use water as a solvent, as was done by Pan et al. in 2011. They reported that the synthesis of ZIF-8 in water solvent at room temperature, but with a ratio of $\mathrm{Zn}^{2+}$ large enough: methylimidazole, namely 1:70. The resulting ZIF-8 has a surface area of $1079 \mathrm{~m}^{2} / \mathrm{g}$.

\section{METHODS}

\section{A. Materials}

Zinc nitrate butahydrate $\left(\mathrm{Zn}\left(\mathrm{NO}_{3}\right)_{2} .4 \mathrm{H}_{2} \mathrm{O}, 98 \%\right)$, Methanol (MeOH, 98\%) and 2-methyl imidazole (MeIM, 99\%) and iron(II) sulfate heptahydrate $\left(\mathrm{FeSO}_{4} .7 \mathrm{H}_{2} \mathrm{O}, 98 \%\right)$. All of matherials were obtained from Sigma Aldrich and use without further purification.

\section{B. Synthesis of Fe-ZIF-8 Crystals}

The Fe-ZIF-8 synthesis method used in this study is a combination of methods $[10,11]$. The molar ratio used was 1 : 4: 175 for $\mathrm{Zn}^{2+}$ : methylimidazole: $\mathrm{MeOH}$. The $\mathrm{FeSO}_{4} \cdot 7 \mathrm{H}_{2} \mathrm{O}$ added in the synthesis was $5 \%$. Zinc nitrate solution was prepared by dissolving $2.85 \mathrm{mmol} \mathrm{Zn}\left(\mathrm{NO}_{3}\right)_{2} .4 \mathrm{H}_{2} \mathrm{O}$ and 0.15 mmol $\mathrm{FeSO}_{4} .7 \mathrm{H}_{2} \mathrm{O}$ in $526 \mathrm{mmol}$ methanol (solution A). Next, $12.0 \mathrm{mmol}$ of 2-methylimidazole would be dissolved into 526 mmol of methanol (solution B). The two solutions were put in a closed glass bottle, stirred for 24 hours at room temperature, the solution obtained were then centrifuged and washed with methanol. Washing were carried out up to three times and then 
the precipitate obtained were then dried for 24 hours at a temperature of $120^{\circ} \mathrm{C}$. In the second synthesis used the filtrate produced from the previous synthesis as a solvent. All forms of treatment were carried out the same as the previous synthesis. The resulting material was given the notation Fe-ZIF-8.1. The repetition was done twice and the next material was given the notation Fe-ZIF-8.2.

\section{Characterization}

The characterization of X-ray Diffraction (XRD) studies were carried out with an X-ray diffraction (XPERT MPD, 30 $\mathrm{mA}, 40 \mathrm{kV}$ ) with $\mathrm{Cu}$ radiation. The XRD patterns were recorded for $2 \theta^{\circ}$ between $5^{\circ}$ and $55^{\circ}$ and compared with XRD pattern of ZIF-8 synthesized by Gross et al., 2012. FTIR characterization were done on Shimadzu FTIR spectrometer with resolution of $1 \mathrm{~cm}^{-1}$. $\mathrm{KBr}$ pellets of the samples were analyzed in range $4000-400 \mathrm{~cm}^{-1}$.

\section{RESULTS AND DISCUSSION}

\section{A. Synthesis of Fe-ZIF-8 by Using Filtrate}

Zeolitic Imidazole Framework (ZIF-8) in this study was synthesized by adding $\mathrm{FeSO}_{4} .7 \mathrm{H}_{2} \mathrm{O}$ at room temperature and filtrate as a solvent. Room temperature was chosen as one of the synthesis methods as an effort to create a material synthesis with an environmentally friendly method. In addition, the use of filtrate as a solvent is also a method alternative of green solvent.

The zinc nitrate tetrahydrate $\left(\mathrm{Zn}\left(\mathrm{NO}_{3}\right)_{2} .4 \mathrm{H}_{2} \mathrm{O}, 98 \%\right)$ as a central metal source was added to a solution of methyl imidazole and methanol. Methyl imidazole used in this study was an organic compound that acts as a ligand provider in the skeleton structure. Meanwhile, $\mathrm{FeSO}_{4} .7 \mathrm{H}_{2} \mathrm{O}$ as an additional metal was only used $5 \%$ of the main metal source. The reaction mixture was then stirred using a magnetic stirrer for 24 hours to obtain a homogeneous solution. Next, the reaction mixture was left to stand for 24 hours for the complete reaction. It is in line with Cravillon et al. [2] reporting that the maximum time needed in the synthesis of ZIF-8 using solvent methanol as a is 24 hours. After being idled, two layers were formed, the lower layer was a light brown sediment, while the upper layer was a clear, colorless liquid. This brown solid is probably the result of the reaction of $\mathrm{Fe}^{+2}$ metal and ligands MeIM.

In the case of porous materials, the solvent molecules that fill the pore cavities must be removed first to activate the material to accommodate new molecules. The solvent molecules can be removed by washing using methanol which is a solvent volatile. Finally, the synthesis of Fe-ZIF-8 was accomplished by emptying the pores of crystals from the solvent of methanol by heating at $120^{\circ} \mathrm{C}$ for 24 hours. The material obtained was Fe-ZIF-8; Fe-ZIF-8.1 and Fe-ZIF-8.2 are light brown in color while ZIF-8 without doping is white. The brown color resulting from the material indicates that $\mathrm{Fe}^{2+}$ has been doped on ZIF-8.

\section{B. Characterization of Fe-ZIF-8 by using Filtrat}

Figure 1 shows the XRD pattern of Fe-ZIF-8.1 material which was synthesized at room temperature using a filtrate as a solvent. The X-ray diffraction pattern has characteristic peaks that appear at $2 \theta$, namely, $10.34^{\circ} ; 12.64^{\circ} ; 16.28^{\circ}$ and $17.93^{\circ}$ are similar to the XRD pattern of simulated ZIF-8 crystal structure. ZIF- 8 has characteristic peaks appearing at $10.34^{\circ}$; $12.72^{\circ} ; 16.36^{\circ}$; and $17.98^{\circ}$ [12]. This pattern shows that the sample has a pure ZIF-8 phase. However, in the second repetition of the synthesis Fe-8.2-ZIF, there were similarities with the diffraction pattern of ZIF-8 simulation or with Fe-8.1ZIF previously synthesized.

Based on the analysis using XRD Fe-ZIF-8.1, it has a high intensity peak at an angle of $2 \theta=12.64^{\circ}$, followed by a moderate intensity at a peak of $17.93^{\circ}$ and a low intensity peak at an angle of $2 \theta=16.28^{\circ}$ and $10.34^{\circ}$. Based on the XRD pattern obtained from the Fe-ZIF- 8 material, there are several other peaks that appear which indicate the presence of $\mathrm{Zn}(\mathrm{OH})\left(\mathrm{NO}_{3}\right)\left(\mathrm{H}_{2} \mathrm{O}\right)$ in the resulting material. According to Bao et al [3], the difference in the molar ratio of MeIM/ $\mathrm{Zn}^{2+}$ has an important influence on the crystallization process of ZIF-8. The initial $\mathrm{pH}$ value of a solution is directly proportional to the increase in the molar ratio $\mathrm{MeIM} / \mathrm{Zn}^{2+}$. Changes in $\mathrm{pH}$ value can also change the self-formation process of ZIF-8 due to interactions with the imino hydrogen-atom in MeIM.

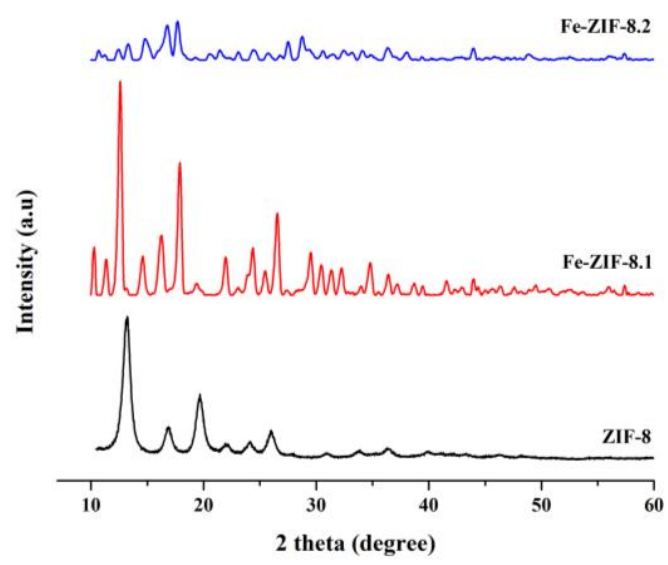

Fig. 1. X-ray diffraction pattern of the sample Fe-ZIF-8 synthesized.

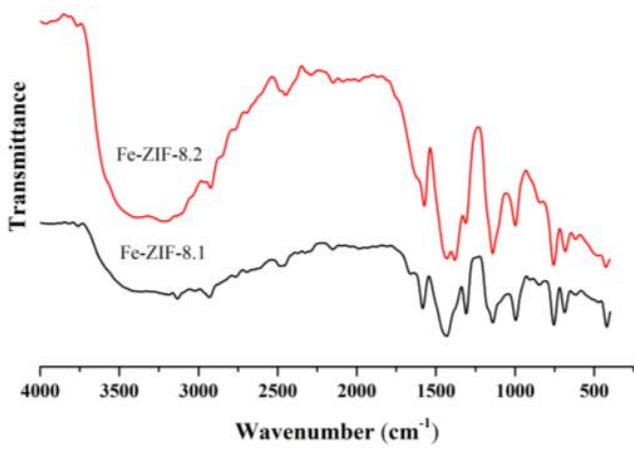

Fig. 2. FTIR spectra of Fe-ZIF-8.1 and Fe-ZIF-8.2. 
Figure 2 shows the FTIR spectra of Fe-ZIF-8.1 and Fe-ZIF8.2 synthesized using a filtrate. Almost all FTIR absorption bands produced correspond to the previously reported ZIF-8 FTIR absorption bands [13]. For example, there is an absorption band in the area of wave number $420 \mathrm{~cm}^{-1}$. It indicates a stretching vibration in $\mathrm{Zn}-\mathrm{N}$, meaning that in the sample a bond is formed between $\mathrm{Zn}$ and $\mathrm{N}$ in imidazole. Furthermore, there is an absorption band in the area of wave number $1582 \mathrm{~cm}^{-1}$ with a weak intensity, which is the bending vibration of $\mathrm{C}=\mathrm{N}$. The weak absorption band at the wave number indicates that the $\mathrm{Zn}$ atom has bonded with the $\mathrm{N}$ atom of the imidazole ligand, so that the number of $\mathrm{C}=\mathrm{N}$ bonds is getting smaller. This is also supported by the presence of a $\mathrm{CN}$ aromatic ulcer absorption band in the wavenumber region of around $1140 \mathrm{~cm}^{-1}$ and $\mathrm{CN}$ bending vibration in the area of wave number $996 \mathrm{~cm}-1$. Next, there is an absorption band at 3132 and $2930 \mathrm{~cm}^{-1}$. It indicates that there is an absorption band from the $\mathrm{CH}$ spbonds ${ }^{2}$ and $\mathrm{CH} \mathrm{sp} \mathrm{sp}^{3}$ on the imidazole ligand [14]. The FTIR analysis result data supports XRD analysis data which shows that Fe-ZIF-8.1 has been successfully synthesized.

\section{CONCLUSION}

Zeolitic Imidazole Framework-8 (ZIF-8) which was doped with $\mathrm{Fe}$ (Fe-ZIF-8.1) has been successfully synthesized by utilizing waste filtrate as a solvent. The results of the characterization show that there is a structural similarity between ZIF-8 which was synthesized using pure solvent and Fe-ZIF-8.1 which was synthesized using the previous waste filtrate. Utilization of the synthesized filtrate used as a solvent in the synthesis of the next material is one way to minimize the use of organic solvents as a solvent to create green chemistry in the synthesis of inorganic materials in particular.

\section{ACKNOWLEDGMENT}

This research funded by Ministry of Research and Technology, National Research and Innovation Agency of the Republic of Indonesia. We would like to thank several institutions including Basic Laboratory of Samudra University, Chemistry Laboratory of Syiah Kuala University.

\section{REFERENCES}

[1] J.C. Tan, T.D. Bennett, and A.K. Cheetham, "Chemical structure, network topology, and porosity effects on the mechanical properties of Zeolitic Imidazolate Frameworks.” PNAS, vol. 107, no. 22, p. 9938 2010

[2] J. Cravillon, S. Münzer, S.J. Lohmeier, A. Feldhoff, J. Huber and M Wiebcke, "Rapid Room-Temperature Synthesis and Characterization of Nanocrystals of a Prototypical Zeolitic Imidazolate Framework." America Chemistry Society, vol. 21, pp. 1410-1412, 2009.

[3] Q. Bao, Y. lou, T. Xiang, and J. Chen, "Rapid Synthesis of Zeolitic Imidazolate Framework-8 (ZIF-8) in Aqueous Solution Via Microwave Irradiation.” Inorganic Chemistry Communication, vol. 37, pp. 170-173, 2013.

[4] A. Phan, C.J. Doonan, R.F.J. Urebe, C.B. Knolbler, M. O'Keeffe, and O.M. Yaghi, "Synthesis, Structure, and Carbon Dioxide Capture Properties of Zeolitic Imidazolate Frameworks." Accounts of Chemical Research, vol. 43, pp. 58-67, 2010.

[5] Y. Pan, Y. Liu, G. Zeng, L. Zhao, and Z. Lai, "Rapid Synthesis of Zeolitic Imidazolate Framework-8 (ZIF-8) Nanocrystals in an Aqueous System." The Royal Society of Chemistry, vol. 47, pp. 2017-2073, 2011.

[6] A.F.P. Ferreira, M.C. Mittelmeijer-Hazeleger, M.A. Granato, V.F.D Martins, A.E. Rodrigues, and G. Rothenberg, "Sieving Di-Branched from Mono-Branched and Linear Alkanes Using ZIF-8: Experimental Proof and Theoretical Explanation." Physic Chemistry, vol. 15, pp 8795-8804, 2013

[7] N.A.H.M. Nordin, A.F. Ismail, A. Mustafa, P.S. Goh, D. Rana, and T Matsuura, "Aqueous Room Temperature Synthesis of Zeolitic Imidazole Framework 8 (ZIF-8) with Various Concentrations of Triethylamine." The Royal Society of Chemistry, vol. 4, pp. 33292-33300, 2014.

[8] J.M. Yang, Q. Liu, and W.Y. Sun, "Shape and Size Control and Gas Adsorption of Ni(II)- Doped MOF-5 Nano/Microcrystals.” Microporous and Mesoporous Material, vol. 190, pp. 26-31, 2014.

[9] J.A. Botas, G. Calleja, M. Sanchez, and M.G. Orcajo, "Effect of Zn/Co ratio in MOF-74 type materials containing exposed metal sites on thei hydrogen adsorption behaviour and on their band gap energy." Hydrogen Energy, vol. 36, pp. 10834-10844, 2011.

[10] M.T. Thanh, T.V. Thie, V.T. Chau, P.D. Du, N.P. Hung, and D.Q. Khieu "Synthesis of Iron Doped Zeolite Imidazolate Framework-8 and Its Remazol Deep Black RGB Dye Adsorption Ability." Hindawi Journal of Chemistry, pp. 1-18, 2017.

[11] N.K. Demir, B. Topuz, L. Yilmaz, and H. Kalipcilar, "Synthesis of ZIF8 from Recycled Mother Liquors." Microporous and Mesoporous Material, vol. 198, pp. 291-300, 2014.

[12] A.F. Gross, E. Sherma, and J.J. Vajo, "The Royal Society of Chemistry." Vol. 41, pp. 5458-5460, 2012.

[13] Z. Zhang, S. Xian, H. Xi, H. Wang, and Z. Li, Chem Eng Sci, pp. 664878-4888, 2011.

[14] M.J.C. Ordonez, K.J. Balkus, J.P. Ferraris, and I.H. Musselman, "Molekular Sieving Realized with ZIF-8/Matrimid Mixed-Matrix Membranes.” Journal of Membrane Science, vol. 361, pp. 28-37, 2010. 